\title{
SISTEMA PARA CONTROLE DE VELOCIDADE DE MOTOR BRUSHLESS EM MALHA
}

\author{
FECHADA
}

\section{CLOSED LOOP SPEED CONTROL SYSTEM FOR BRUSHLESS MOTORS}

Marcio Santin ${ }^{1}$

Eduardo Bueno ${ }^{2}$

\section{RESUMO}

Esse trabalho apresenta o estudo e a implementação de um sistema de controle de velocidade de motores Brushless em malha fechada. Esses motores estão sendo amplamente utilizados em diversos setores da indústria devido às vantagens perante os demais modelos de motor de corrente contínua. O foco desse estudo são os motores Brushless que possuem realimentação por sensores de efeito Hall, que além de informar a posição do rotor, serve como feedback de velocidade do motor. Para implementar o sistema de controle utiliza-se um microcontrolador da família dsPIC da Microchip ${ }^{\circledR}$. Para o controle de velocidade foi incorporado um controle PI (Proporcional-Integral). O circuito de potência é uma ponte inversora trifásica, composta principalmente por acopladores ópticos e MOSFETs de potência que será modulada por PWM (Pulse Width Modulation) com seus disparos alternando a cada $120^{\circ}$ elétricos obedecendo às características do motor utilizado.

Palavras-Chave: Motor Brushless. Controle de Velocidade. Controle PI.

\section{ABSTRACT}

This research work shows the study and implementation of a speed control system for Brushless motors in closed loop. These motors are being widely used in several industry sectors due to the advantages over the other models of DC motors. The focus of this study are the Brushless motors which have their feedback by Hall effect sensors, that, detect the rotor magnet position, can also as a motor speed feedback. For implementing the control system, a dsPIC microcontroller by Microchip ${ }^{\circledR}$ is used,

1 Acadêmico do Curso de Engenharia Elétrica. Universidade Alto Vale do Rio do Peixe. Email:santinmr@hotmail.com.

2 Professor Mestre do Curso de Engenharia Elétrica. Universidade Alto Vale do Rio do Peixe. bueno@uniarp.edu.br. 
once it has dedicated functions for motor control. For speed control it has been incorporated a PI (proportional - Integral) control. The power circuit is a three-phase inverter bridge, consisting maily of optocouplers and power mosfet which will be modulated by a PWM (Pulse width Modulation) with its alternate duty cycle every 120 eletrical degrees, obeying the characteristics of the motor in use.

Keywords: Brushless DC motors. speed controller. PI controller.

\section{INTRODUÇÃO}

O motor Brushless é desenvolvido com base em motores DC com escovas. A teoria de máquina moderna foi estabelecida quando Faraday descobriu o fenômeno de indução eletromagnética em 1831. O primeiro motor DC nasceu na década de 1840. Comparando as desvantagens dos motores tradicionais na década de 1930, alguns estudiosos começaram a desenvolver motores sem escovas em que foi implementada à comutação eletrônica, no entanto, naquela época, os dispositivos eletrônicos de potência ainda estavam em fase inicial de desenvolvimento, os estudiosos não conseguiram encontrar um dispositivo de comutação apropriado. Este tipo de motor, pouco confiável e de baixa eficiência, foi utilizado apenas em laboratório, ao invés de ser popularizado. No ano de 1955 Harrison e Rye fizeram o primeiro pedido de patente para um circuito de comutação com tiristores para substituir o equipamento mecânico de comutação (XIA, 2012). Desde a sua invenção no século XIX os motores elétricos estão em constante evolução, sempre em busca do aperfeiçoamento de sua eficiência e do aumento de sua vida útil. Dentre os vários modelos existentes, o motor de corrente contínua sem escovas (Brushless) tem ganhado maior destaque e importância frente aos demais motores de corrente contínua convencionais.

Segundo Yedamale (2003), os motores Brushless se popularizaram rapidamente e estão sendo amplamente utilizados em diversos setores da indústria. As principais vantagens dos motores DC Brushless são: sua eficiência, menor peso, menor custo de manutenção, menor ruído e trabalham em grandes faixas de velocidade.

Nos últimos anos a busca por motores elétricos mais eficientes tem aumentado significativamente, essa melhora no rendimento dos motores está diretamente ligada ao projeto do motor e ao sistema de controle que será utilizado 
(YASHVANT, 2006).

O controle de velocidade desempenha um papel importante no controle do motor. Os principais métodos de controle empregados são de malha aberta e malha fechada. Devido a sua simplicidade, robustez, confiabilidade e fácil ajuste, o controle PID durante anos vem sendo amplamente utilizado em sistemas industriais. $O$ controlador PID calcula o desvio entre o valor de referência e o valor real do processo, onde KP é o ganho proporcional, TI é o tempo de integração e TD é o tempo diferencial, porém, nem todos os controladores possuem os três termos, dentre eles são: controlador proporcional, o controlador proporcional-integral, o controlador proporcional-derivativo, dentre outros. Para controle de motores Brushless geralmente utiliza-se o controle proporcional-integral (XIA, 2012).

Para Cooper (2015), o controle PI possui menor complexidade que os demais tipos de controle, por isso é o algoritmo mais utilizado no controle de processos. Diferentes fabricantes utilizam basicamente variações do mesmo algoritmo de controle PI.

Segundo Ogata (2000), um sistema de controle em malha fechada é todo sistema que possui um sinal de feedback do processo que pode ser o próprio sinal de saída ou uma função dele, tornando o sistema menos sensível a perturbações.

Pode-se fazer o controle de velocidade em malha fechada básico utilizando os sensores Hall do próprio motor, caso seja necessário fazer um controle preciso recomenda-se utilizar um encoder externo (YEDAMALE, 2003).

A maioria dos motores Brushless possui enrolamentos trifásicos conectados internamente em estrela, porém, somente duas fases são energizadas a cada ciclo. O segredo para o acionamento do motor Brushless é detectar a posição do rotor e depois energizar as fases que produzirão o maior torque no sentido em que se deseja girar. A maneira mais fácil de saber a posição do rotor e determinar quais fases serão energizadas é através dos sensores Hall. Quando o pólo norte passar pelo sensor Hall, sua saída será alta ou estado 1, quando passar o pólo sul, a saída será baixa ou estado 0 (BROWN, 2011).

Segundo Yashvant (2006), uma sequência de comutação de seis etapas é usada para direcionar a corrente e produzir torque. Para fazer o acionamento das fases do motor utiliza-se uma ponte trifásica composta por seis chaves controladas, que, dependendo da potência e tensão do motor pode ser confeccionada com 
MOSFETs, IGBTs ou por transistores bipolares.

Baseados nessa sequência de acionamentos das fases do motor podemos afirmar que o motor irá girar em velocidade nominal. Caso seja necessário variar a velocidade, podemos conectar um potenciômetro no microcontrolador para servir de referência. Para variar a velocidade os sinais que acionam os MOSFETs devem ser modulados por PWM (Pulse Width Modulation) em uma frequência de no mínimo dez vezes maior que a frequência máxima do motor (YEDAMALE, 2003).

Este trabalho tem por finalidade o desenvolvimento de um sistema de controle de velocidade para motores Brushless que possa ser utilizado em laboratório, como fonte de pesquisa acadêmica.

\section{DESCRIÇÃO DO SISTEMA DESENVOLVIDO}

O sistema desenvolvido nesse trabalho compreende um sistema de controle de velocidade em malha fechada utilizando como feedback três sensores Hall instalados no motor com uma defasagem de 120 graus elétricos.

Esse sistema foi desenvolvido basicamente utilizando um microcontrolador da família dsPIC da Microchip e uma ponte H trifásica com MOSFETs.

\section{DESENVOLVIMENTO DO SOFTWARE}

Para o desenvolvimento da rotina de controle em malha fechada utilizouse a linguagem de programação $C$. A ferramenta de desenvolvimento escolhida foi o

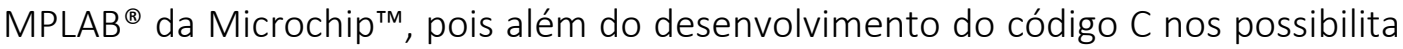
efetuar simulações, compilar e gravar o programa na memória do microcontrolador. O funcionamento do software dá-se da seguinte forma:

a) Após a energização do circuito o microcontrolador executa uma rotina de configuração das portas de I/O (entradas e saídas) e inicialização de variáveis internas.

b) Ao ser pressionado o botão liga verifica-se o sentido de giro horário ou anti-horário que é selecionado através de um botão, e habilita-se a execução das interrupções externas "CN" (Change Notification Inputs) que são disparadas através do acionamento dos sensores Hall que tem a função de determinar a posição atual 
do rotor e determinar qual será a sequência de acionamento das fases do motor.

c) Depois de definida a sequência de acionamento a interrupção "ACD" (Analog-Digital Conversion Complete) calcula a velocidade atual do motor utilizando o sinal recebido do sensor Hall "A", calcula os ganhos, ajusta o ciclo dos PWMs para manter uma velocidade constante e permanece nesse loop até que o comando liga seja pressionado outra vez.

d) Para efetuar a parada do motor deve-se pressionar o botão liga, nesse instante as interrupções e os PWMs são desabilitados fazendo com que o motor pare de girar.

\section{DESENVOLVIMENTO DO CIRCUITO}

Para o desenvolvimento e simulação do circuito eletrônico optou-se pelo software Proteus ${ }^{\circledR}$ da Labcenter ${ }^{\mathrm{TM}}$, pois propicia a simulação completa do circuito de controle e potência, bem como o desenvolvimento do layout e da placa do circuito. Para esse desenvolvimento seguiu-se os seguintes passos:

No primeiro passo verificou-se a sequência e o nível dos sinais dos sensores Hall. No próximo passo verificou-se os sinais das saídas PWMs do microcontrolador e os sinais de saída dos MOSFETs.

O último passo foi conectar o motor ao circuito realizando assim os ajustes dos ganhos do controle PI implementado. A figura 1 mostra as saídas PWM 1 e 2 durante simulação.

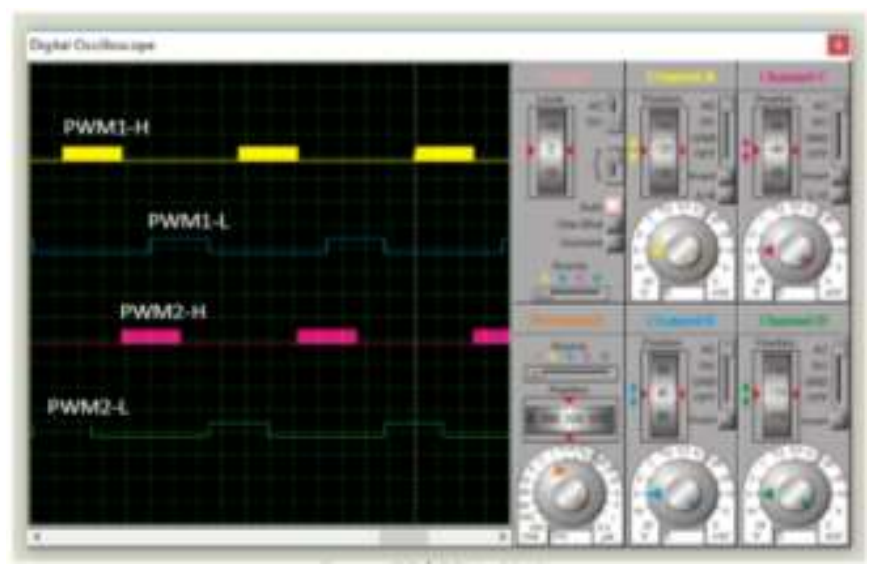

Figura 1. Saídas PWM 1 e 2 durante simulação em software 


\section{APRESENTAÇÃO E RESULTADOS}

O protótipo é composto basicamente por um microcontrolador e uma ponte $\mathrm{H}$ trifásica. Por se tratar de um protótipo para uso didático optou-se pela fabricação de duas placas independentes, uma de controle e outra de potência, pois possibilita aos usuários a fácil implementação de um circuito de potência que atenda a demanda requerida para cada projeto específico apenas substituindo a ponte $\mathrm{H}$. Com relação ao sistema de controle foi disponibilizado ao usuário botões com as seguintes funções: reset do microcontrolador, comando liga/desliga motor, comando de reversão e um potenciômetro para ajuste de velocidade, bem como um conector para que seja possível realizar a programação de microcontrolador sem a necessidade de removê-lo do circuito. Outro diferencial é a possibilidade de selecionar o tipo de sinal recebido dos sensores Hall que podem ser do tipo senoidal ou trem de pulso. Na Figura 2 e 3 são apresentadas as placas de controle e potência.

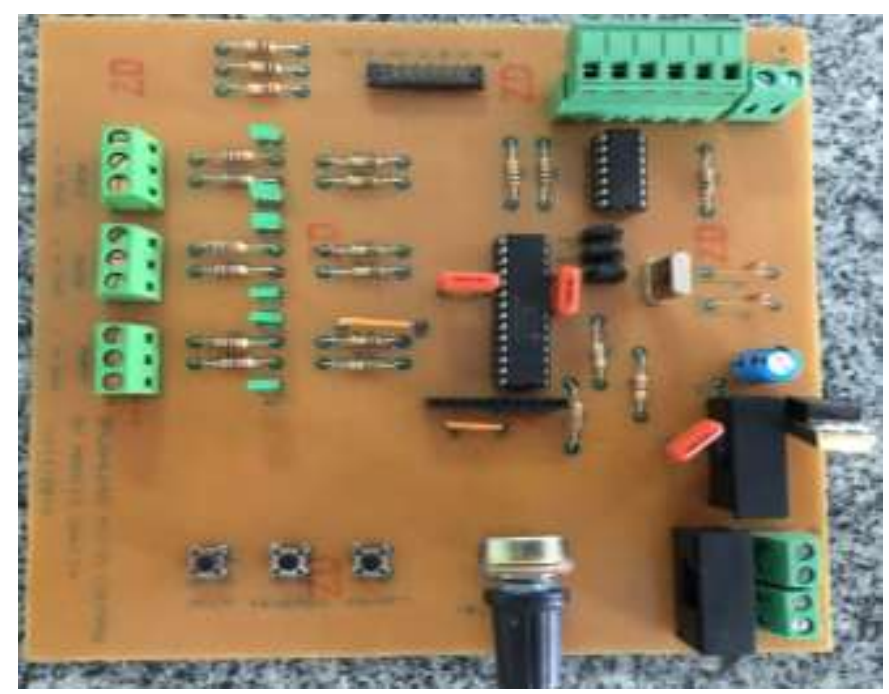

Figura 2. Placa de controle 


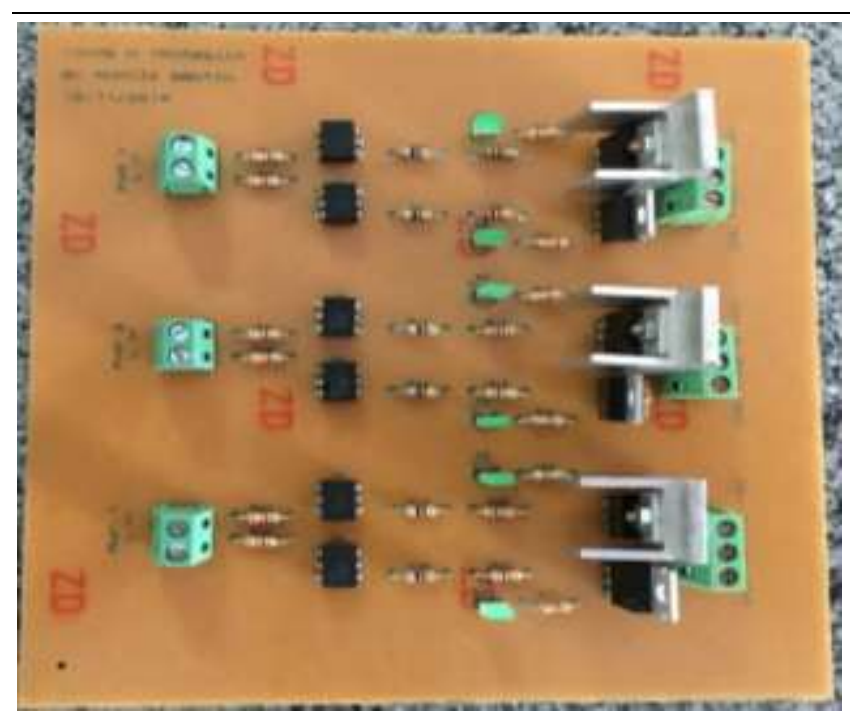

Figura 3. Placa de potência

Pode-se verificar através das medições realizadas em bancada que temos uma variação nos níveis de tensão fornecidos ao motor devido a ação do algoritmo de controle, diferentemente do que acontece com um controle em malha aberta, onde o nível de tensão varia proporcionalmente a velocidade desejada. A resposta do sistema depende dos valores ajustados nos ganhos PI (proporcional e integral) da malha de controle. Quanto maior os ganhos, mais rápida será a resposta, porém, um ganho elevado pode ocasionar variações bruscas de velocidade. Na Tabela 1 apresenta-se dados de velocidade desejada versus velocidade real obtidos em simulação. 
Tabela 1. Velocidade desejada versus atual

\begin{tabular}{l|l|l}
\hline Medição & $\begin{array}{l}\text { Velocidade } \\
\text { desejada } \\
\text { (RPM) }\end{array}$ & $\begin{array}{l}\text { Velocidade } \\
\text { atual (RPM) }\end{array}$ \\
\hline 1 & 328 & 326 \\
\hline 2 & 500 & 499 \\
\hline 3 & 680 & 678 \\
\hline 4 & 770 & 771 \\
\hline 5 & 900 & 901 \\
\hline 6 & 1000 & 998 \\
\hline 7 & 1130 & 1129 \\
\hline 8 & 1250 & 1252 \\
\hline 9 & 1320 & 1319 \\
\hline 10 & 1430 & 1429 \\
\hline
\end{tabular}

\section{CONCLUSÕES}

Ao finalizar o projeto realizou-se ensaios que comprovaram sua funcionalidade e por se tratar de um protótipo para uso didático atenderam às expectativas, deste modo, apresenta-se várias possibilidades para futuras implementações no circuito como medição de corrente nas fases do motor e uma interface gráfica que possibilite efetuar ajustes de parâmetros do controle que hoje só podem ser alterados via software.

\section{REFERÊNCIAS}

XIA, Chang-liang. Permanent magnet brushless DC motor drives and controls. John Wiley \& Sons Singapore Pte. Ltd, 2012.

YEDAMALE, Padmaraja. AN885 - Brushless DC (BLDC) Motor Fundamentals. 2003. Disponível em:

<http://ww1.microchip.com/downloads/en/AppNotes/00885a.pdf>. Acesso em 07 mar 2018.

YASHVANT Jani. Implementing Embedded Speed Control for Brushless DC Motors. Renesas Technology America, Inc. 27 dez 2006. Disponível em: 
$<$ https://www.embedded.com/design/mcus-processorsandsocs/4006773/Implementing-Embedded-Speed-Control-for-Brushless-DCMotorsPart-1>. Acesso em 25 abr 2018.

COOPER, Douglas. Integral Action and PI Control. 07 abr 2015. Disponível em: < https://controlguru.com/integral-action-and-pi-control/>. Acesso em 11 set 2018.

OGATA, Katsuhiko. Engenharia de Controle Moderno. 3. ed. Rio de janeiro: LTC Editora, 2000.

BROWN, Ward. AN857 - Brushless DC Motor Control Made Easy. 2011. Disponível em: <http://ww1.microchip.com/downloads/en/AppNotes/00857B.pdf>. Acesso em 25 abr 2018. 